\title{
Enabling Advanced Wind-Tunnel Research Methods Using the NASA Langley 12-Foot Low Speed Tunnel
}

\author{
Ronald C. Busan ${ }^{1}$, Paul M. Rothhaar ${ }^{2}$, Mark A. Croom ${ }^{3}$, and Patrick C. Murphy. ${ }^{4}$ \\ NASA Langley Research Center, Hampton, Virginia, 23681-2199 \\ Sue B. Grafton ${ }^{5}$ \\ Analytical Mechanics Associates, Inc., Hampton, Virginia, 23681-21999 \\ and \\ Anthony W. O-Neal ${ }^{6}$ \\ VIGYAN, Inc., Hampton, Virginia, 23681-21999
}

\begin{abstract}
Design of Experiment (DOE) testing methods were used to gather wind tunnel data characterizing the aerodynamic and propulsion forces and moments acting on a complex vehicle configuration with 10 motor-driven propellers, 9 control surfaces, a tilt wing, and a tilt tail. This paper describes the potential benefits and practical implications of using DOE methods for wind tunnel testing - with an emphasis on describing how it can affect model hardware, facility hardware, and software for control and data acquisition. With up to 23 independent variables (19 model \& 2 tunnel) for some vehicle configurations, this recent test also provides an excellent example of using DOE methods to assess critical coupling effects in a reasonable timeframe for complex vehicle configurations. Results for an exploratory test using conventional angle of attack sweeps to assess aerodynamic hysteresis is summarized, and DOE results are presented for an exploratory test used to set the data sampling time for the overall test. DOE results are also shown for one production test characterizing normal force in the Cruise mode for the vehicle.
\end{abstract}

\section{Nomenclature}

$\begin{array}{ll}D O E & =\text { design of experiment } \\ U A V & =\text { Uninhabited Air Vehicle } \\ V / S T O L & =\text { Vertical or Short Takeoff and Landing } \\ C A D & =\text { computer aided design } \\ D E P & =\text { distributed electric propulsion } \\ O F A T & =\text { one factor at a time } \\ F C D & =\text { face centered design } \\ L S D & =\text { least significant difference } \\ \alpha & =\text { angle-of-attack in degrees } \\ \beta & =\text { sideslip angle in degrees } \\ C N & =\text { normal force coefficient } \\ L: T t i l t & =\text { tail tilt angle in degrees } \\ T E & =\text { trailing edge } \\ L W S 1 & =\text { left wing TE control surface 1 (nearest tip) }\end{array}$

\footnotetext{
${ }^{1}$ Senior Research Engineer, Flight Dynamics Branch, MS 308, Associate Fellow.

${ }^{2}$ Research Engineer, Dynamic Systems \& Control Branch, MS 308.

${ }^{3}$ Senior Research Engineer, Flight Dynamics Branch, MS 308.

${ }_{5}^{4}$ Senior Research Engineer, Dynamic Systems \& Control Branch, MS 308, Associate Fellow.

${ }^{5}$ Senior Research Engineer, Flight Dynamics Branch, MS 308.

${ }^{6}$ Systems Programmer, Flight Dynamics Branch, MS 308.
} 


$\begin{array}{ll}\text { LWS2 } & =\text { left wing TE control surface } 2 \text { (mid span) } \\ \text { LWS3 } & =\text { left wing TE control surface } 3 \text { (nearest root) } \\ \text { RWS4 } & =\text { right wing TE control surface } 4 \text { (nearest root) } \\ \text { RWS5 } & =\text { right wing TE control surface } 5 \text { (mid span) } \\ \text { RWS6 } & =\text { right wing TE control surface } 6 \text { (nearest tip) } \\ \text { LTS1 } & =\text { left tail TE control surface } 1 \\ \text { RTS1 } & =\text { right tail TE control surface } 2 \\ \text { Rud } & =\text { rudder } \\ \text { LWE1 } & =\text { left wingtip motor } \\ R W E 8 & =\text { right wingtip motor } \\ C & =\text { dwell time } \\ C G & =\text { center of gravity } \\ p s f & =\text { pounds per square foot }\end{array}$

\section{Introduction}

A $\mathrm{n}$ ever-expanding set of mission requirements is driving Uninhabited Air Vehicle (UAV) designs to include numerous conventional and unconventional control effectors, distributed propulsion to enable Vertical or Short Takeoff and Landing (V/STOL), and highly-coupled combinations of configuration elements. One such propellerdriven UAV concept, the GL-10 (Fig. 1 and Fig. 2), includes ten electric motors (eight across the wing and two on the horizontal tail) mounted on a variable incidence wing and a variable incidence horizontal tail (each capable of over 90-degrees of rotation), six trailing edge flaps, two elevators, and one rudder on a ventral vertical stabilizer. The GL-10's mission includes precision VTOL operations, as well as forward flight and achieving the requisite transitions between the modes.

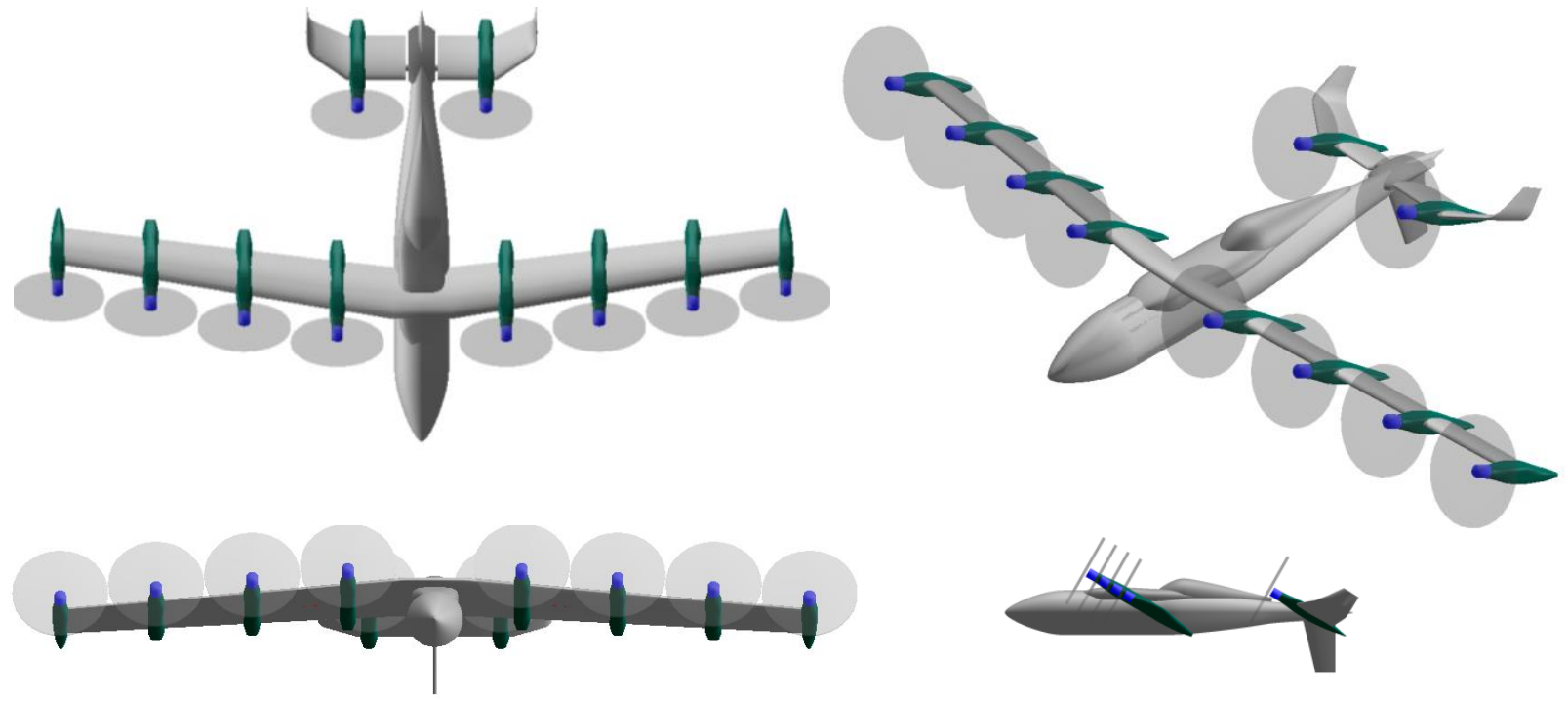

Figure 1. GL-10 CAD Images for Transition Mode Vehicle Configuration 

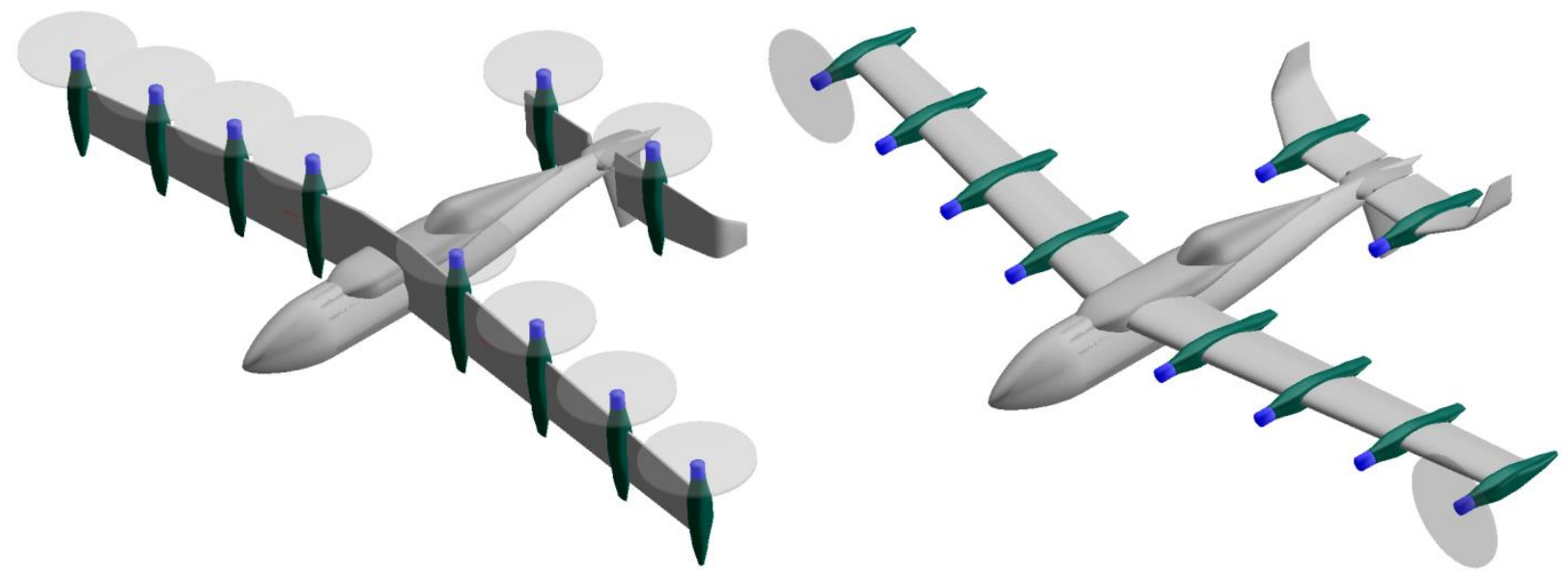

\section{Figure 2. GL-10 CAD Images for Hover and Cruise-Loiter Mode Vehicle Configurations}

Distributed electric propulsion (DEP) is an enabling technology that permits development of complex configurations like the GL-10. From a testing and modelling perspective, DEP generally exacerbates the already complicated propulsion-aerodynamic interaction (PAI) issue that is often present even for single-engine designs; the GL-10 with its ten highly-coupled propeller slipstreams in close proximity to wing and tail lifting surfaces can exploit the PAI not only for improved efficiencies, but also for tailoring the vehicle flight dynamics once the numerous direct and interacted effects are understood. Additional discussion about the benefits of DEP and the project goals for GL-10 can be found in Ref. 1 and the companion paper to this one, Ref. 8.

The full-scale GL-10 UAV design has a wingspan of approximately 20 feet. A 50\% scale prototype flight vehicle airframe has been built, and is currently being outfitted with the required systems for flight testing. To obtain aerodynamic data on the GL-10 configuration, a wind tunnel model was also built and tested in the NASA Langley 12-Foot Low Speed Tunnel. The GL-10 full-scale UAV design, the prototype flight vehicle, and the wind tunnel model all have DEP as a key design feature. Figure 3 provides reference information for both the GL-10 prototype flight vehicle and the wind tunnel model. An important different between the flight vehicle and the wind tunnel model is the propeller configuration. The flight vehicle has 3-bladed folding propellers, whereas the wind tunnel model has 2-bladed fixed propellers. 


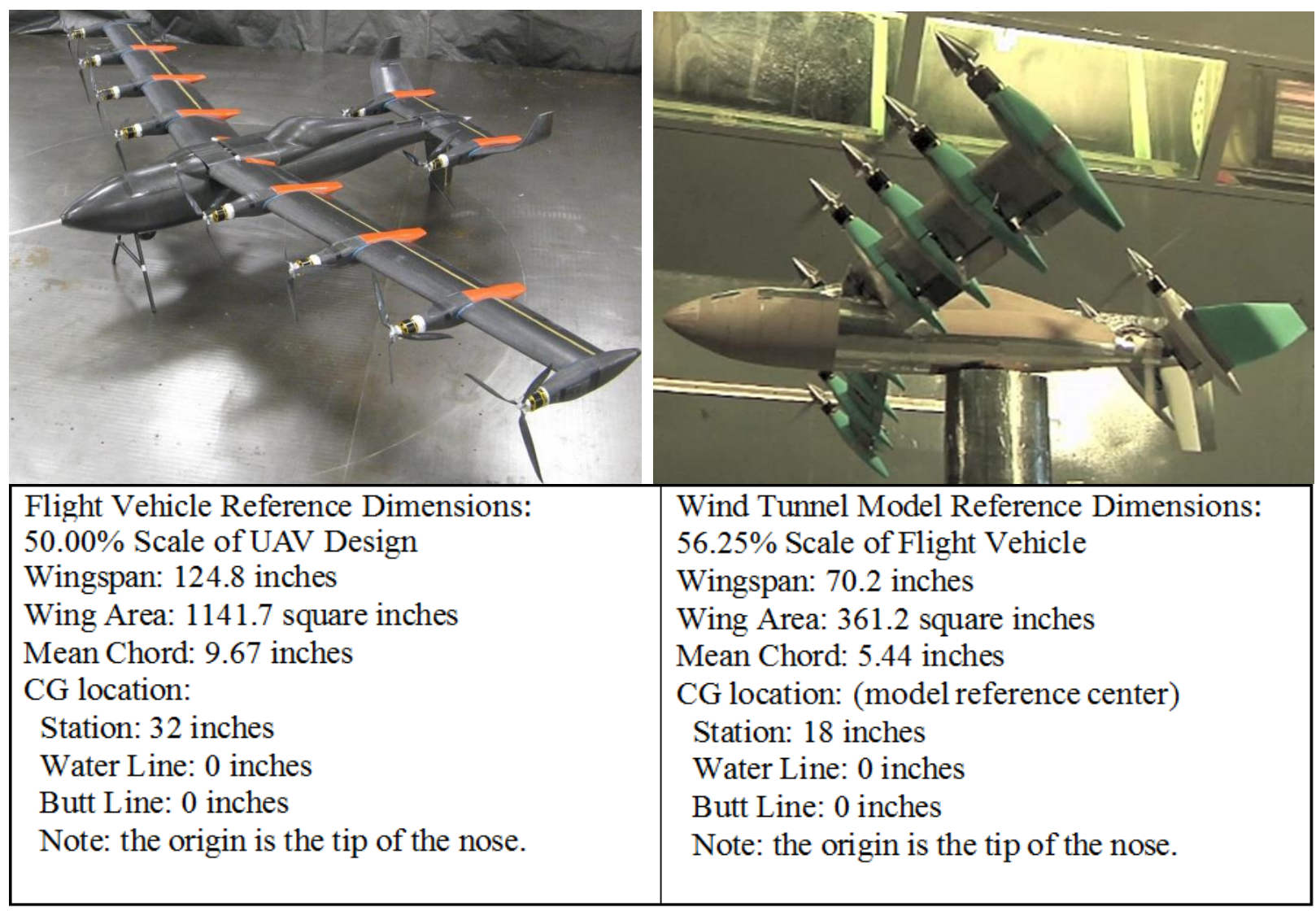

\section{Figure 3. GL-10 Prototype Flight Vehicle and Wind Tunnel Model with Reference Information}

Flight operations of the GL-10 have been broken down into four modes designated as "Transition", "Hover", "Cruise", and "Loiter". Transition covers the entire range of flight operations between Hover (VTOL) and Cruise or Loiter. Cruise and Loiter operations involve the vehicle flying like a conventional airplane. For these modes eight of the motors are turned off and their propellers folded back to reduce drag. Cruise includes the portion of the flight operations where the lift curve slope is fairly linear. Loiter includes flight operations at reduced airspeed and vehicle angles of attack closer to wing stall - generally encompassing a more non-linear portion of the lift curve.

When the GL-10 is operating in Transition mode, 10 motors, 6 wing trailing edge (TE) surfaces, 2 tail TE surfaces, 1 rudder, wing tilt, and tail tilt will all be actively commanded - resulting in a total of 21 independent parameters on the vehicle itself, plus angle of attack and sideslip. For Hover mode the wing tilt and tail tilt are locked at 90 degrees, resulting in a total of 19 independent parameters on the vehicle. Since the Hover testing was done with wind off, angle of attack and sideslip have no impact on the aerodynamics. For Cruise or Loiter mode testing the wing tilt is locked out at zero degrees, and only the two wingtip motors are active. This results in a total of 12 independent vehicle parameters plus angle of attack and sideslip.

Successful flight operations of the GL-10 will require highly augmented control and propulsion mixing through a variety of ever varying conditions during the critical take-off phase and while maneuvering at the target location. Developing the flight system requires extensive knowledge of the air vehicle's performance, stability, and control capabilities. To reduce the risks associated with the initial testing of the prototype flight vehicle the project team decided to develop a simulation model that included critical interaction effects.

Collecting the necessary data using the classic wind-tunnel testing approaches for a complex vehicle with so many independent controls and states of interest would typically require accepting a variety of approximations to reduce the size of the test matrix. For example, control surfaces of like-application are sometimes ganged together to function as one. This approach misses any individual effector influences, and may overlook opportunities for performance optimization or potentially performance-limiting or hazardous stability or control degradations. 
Another method for reducing the test matrix is to examine any particular independent parameter in isolation when mapping out its region of influence. However, this ignores the non-linear coupled response of combined controls and states may similarly miss important attributes to exploit or avoid.

Methods to rapidly evaluate systems with large numbers independent parameters have been maturing over the past 20 years, and generally fall under some form of the name Design of Experiment (DOE). DOE theory gives researchers a methodology for defining experiments that very efficiently determine the effects of input variables, their interactions, and sensitivities of the responses to these inputs. This is done in a statistically rigorous framework that allows optimizations of the designs to produce more robustness with respect to various types of error. For example, in the GL-10 study prediction error characteristics were minimized as part of the experiment design. DOE testing can illuminate important effects and combinations of effects, and can direct limited tunnel resources to place better focus on mission-critical regions having complex non-linear responses. Within the same amount of test occupancy time, DOE testing provides a more complete understanding of the coupled aerodynamics and, therefore, determinations of linearity or non-coupled behavior can be made or avoided based on measured attributes and not solely on assumptions or engineering judgment.

Implementing the DOE testing methodology significantly impacts the model hardware, facility support equipment, tunnel operations, and the software for control, data acquisition, calibration, and analyses. Additional sensors, remote machine-driven controllability, and point-wise data acquisition methods are all required to enable efficient DOE wind-tunnel testing. These and other aspects of applying DOE methods to wind tunnel testing will be discussed in the paper - with an emphasis on highlighting some of the practical differences from more traditional wind tunnel testing techniques. DOE testing in the NASA Langley 12-Foot Low Speed Tunnel was first done in 2002, and examples from several prior tests for simpler vehicle configurations will be used to illustrate some of the lessons-learned, and upgrades to the NASA Langley 12-Foot Low Speed Tunnel that enabled the successful recent testing of the much more complex GL-10 configuration.

\section{GL-10 Wind Tunnel Test Goals and Challenges}

Gaining a basic understanding of the capabilities of the GL-10 configuration is aided by the wind-tunnel testing in the form of direct analyses and by the development of a simulation model that incorporates static aerodynamic properties in the presence of the appropriate propulsive influences. Experimentally determining the performance, stability, and control properties in the 12-Foot Low Speed Tunnel was approached in an abbreviated format using conventional (i.e., non-DOE) methods to ensure the test setup was sound and to gain some overall insights into both the configuration aerodynamics and the testing environment for this particular design.

Goals for the GL-10 simulation model development included characterizing the aerodynamics in a relatively short period of time and at a relatively low cost. A project decision determined that somewhat larger confidence intervals could be tolerated in order to save time and reduce costs. The GL-10 all-electric prototype control system commands 21 control variables, ten of which are propulsion commands. Two of these control signals, wing tilt and tail tilt, significantly change the vehicle's geometric configuration and move the overall center of gravity. The GL10 's flight envelope encompasses a large range of vehicle configurations, airspeeds, and flight attitudes. Including angle of attack and sideslip, 23 variables or factors required investigation in order to properly model this vehicle in the "Transition" mode. For vehicle configurations required for "Cruise/Loiter" and "Hover" testing the total number of variables were 14 and 19 respectively.

Characterizing GL-10 aerodynamics under these requirements presents a number of challenges to conventional methods of test where one factor at a time (OFAT) is varied while holding all other variables constant. The first challenge is that the test time required is prohibitive due to the large number of factors involved. During the final week of the GL-10 DOE test approximately 2000 data points were collected each day. Since OFAT testing would typically have a smaller change in the parameter being varied between successive test points, this theoretically would allow a greater number of test points to be taken in the same amount of time. If 4000 test points per day, and only 5 test points per independent variable is assumed, then for 14 variables the test time required to characterize all the interactions would equal $\left(5^{\wedge} 14\right) / 4000$ days. This would be 4180 years. For 23 variables the time required would be $\left(5^{\wedge} 23\right) / 4000$ days - which is equal to 8 billion years. It is for these reasons that a blindly applied factorial approach encompassing all factors is not pursued in testing of complex systems. Instead, engineering judgment, heritage lessons, or precursor coupling studies help reduce the number of combinations to study for any approach, conventional or DOE. Moreover, DOE methods allow for experimentally-based evaluations of the non-linearities and coupling to occur in a streamlined and statistically rigorous manner.

The second challenge is handling system errors. The conventional method assumes testing apparatus remain stable during the testing period - although this assumption is frequently checked. Corrections for temperature drift, 
zero drift in the measurement systems, etc. can be made - but this typically becomes more difficult for longer test runs. For the GL-10 test, collecting one block of data points typically took hours. Unless data for corrections are being collected over the course of a run, the errors due to the testing system drift can become inseparable from the precision errors.

The solution to these challenges was an experiment designed using DOE theory ${ }^{2}$ that produced extremely efficient, statistically rigorous designs that minimized prediction error. In addition, the experimenter can determine beforehand how much statistical power is available for the design and ensure that sufficient data is collected to achieve the desired statistical performance.

The testing was also facilitated by specific mechanical automation and software capabilities provided in NASA Langley's 12-Foot Wind Tunnel. Facility hardware and software capabilities impacting DOE testing are discussed further in Sections IV and V. These capabilities allowed the basic principles of DOE to be incorporated into the test.

Four principles in DOE theory have a direct bearing on the wind tunnel setup and operational requirements:

1. Orthagonality of Regressors = the assumption that underlies all basic DOE theory. If the modeling functions are multivariate orthogonal functions generated from measured independent variable data, the retained modeling functions can be decomposed into an expansion of ordinary polynomials in the independent variables.

2. Replication $=$ independent and repeated measurements. This provides an internal estimate of system noise and uncertainty.

3. Randomization $=$ randomized input test matrix. This is the basis for the DOE statistical methods, and also helps average out the effects of extraneous factors that may be present.

4. Blocking $=$ a technique to improve precision when making comparisons among the factors of interest, and also reduces variability associated with factors that may influence the experimental response but that are of no direct interest (i.e. Unless a correction is made, temperature differences for testing at different times of day will produce more variability in one run that lasts all day than it will if the same range of static test points is handled with several separate run blocks of test points.)

Effectively these requirements on the test facility are accommodated by three capabilities in the model and test facility: automation, speed, and accurate arbitrary motion. Automation addresses replication and randomization by providing the abilities to command any flow condition or position of the tunnel, test support rig, and all model actuators from a pre-defined test matrix, followed by recording of input commands, achieved set points, and aerodynamic responses. Ideally this information is collected as fast and accurately as possible and then transmitted back in the exact sequence tested to the investigators for analysis. Rapid data acquisition is a key factor that allows a very large number of test points to be collected in a short time. Precision is also helped by incorporating blocking into the experiment design to remove the effects of known sources of error.

\section{GL-10 Experiment Design}

GL-10 parameter characterization was accomplished using three distinctly different types of experiments: exploratory, DOE, and motor-propeller assembly tests without the airframe present. A limited set of exploratory tests were followed by four main DOE tests reflecting the four modes of GL-10 flight operation: (1) Cruise, (2) Loiter, (3) Transition, and (4) Hover. After this a series of tests for single motor-propeller assemblies without the airframe were performed over a large range of incidence angles and power settings. These tests were done for both a wind tunnel model motor-propeller assembly and a prototype flight vehicle motor-propeller assembly. The data from these runs facilitated modeling the propeller effects for a simulation model of the GL-10 prototype flight vehicle.

Exploratory experiments were performed to address the following key issues in advance of the main DOE test:

1. Check for flutter or excessive vibration through the dynamic pressure range, and select the dynamic pressure for the overall test.

2. Determine the presence and severity of aerodynamic hysteresis effects.

3. Determine if vortilons are required.

4. Determine the minimum duration of data samples required.

5. Obtain representative samples of OFAT data for future comparison with DOE results.

6. Determine the degree and location of aerodynamic nonlinearities.

Conventional static test sweeps over the angle of attack and sideslip ranges at increasing dynamic pressures were used to check for the presence of flutter or any other undesirable vibration, and.to assess data quality (measured load 
levels in comparison to signal noise). No flutter or vibration problems were found, and a test dynamic pressure of 3.5 psf was selected based on data quality and tunnel run time considerations. (Running at higher dynamic pressures causes the tunnel drive motor to gradually heat up, potentially limiting the continuous run time available. At a dynamic pressure of 3.5 psf, the GL-10 testing never had to be halted to allow the system to cool down.)

The conventional static test sweeps highlighted the presence and severity of any static aerodynamic hysteresis effects. These data led to a decision that static hysteresis effects, although present in some regions, did not need to be included in the aerodynamic characterization of the relationships between variables. Consequently the main DOE experiment was not designed to incorporate those characteristics as doing so would significantly increase the required test time; the resulting slightly larger measurement errors were accepted up front.

Figure 4 shows normal force for angle of attack sweeps from -8 to 30 degrees and from 30 to -8 degrees for sideslip angles of $-5,0$, and +5 degrees. The plot at a sideslip angle of zero degrees in Fig. 5 shows that the aerodynamic hysteresis for this GL-10 vehicle configuration is very small.

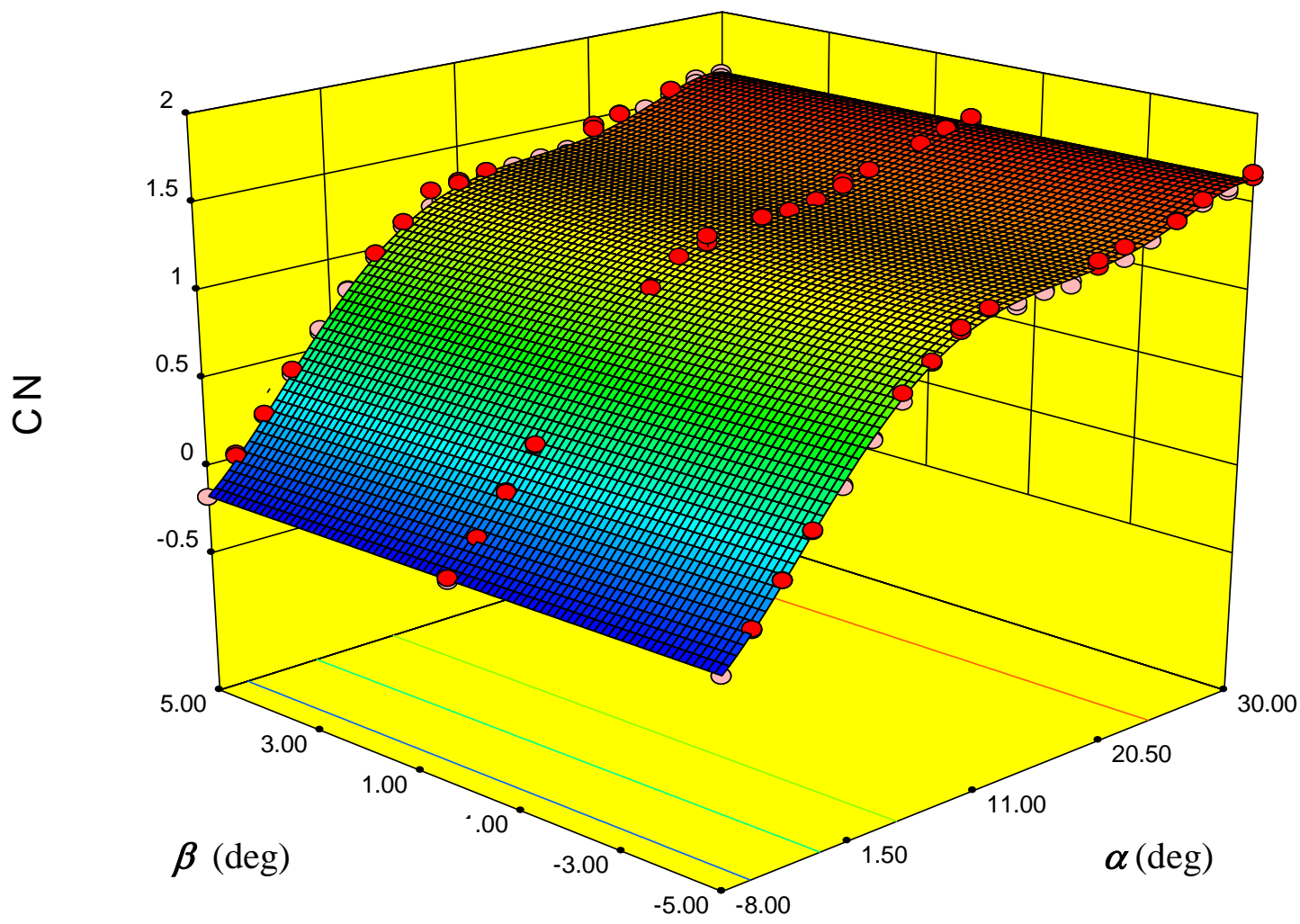

Figure 4. Normal Force as a Function of Angle of Attack for Three Sideslip Angles - GL-10 with Motors Off and Control Surfaces, Wing Tilt, and Tail Tilt All Set to Zero Degrees 


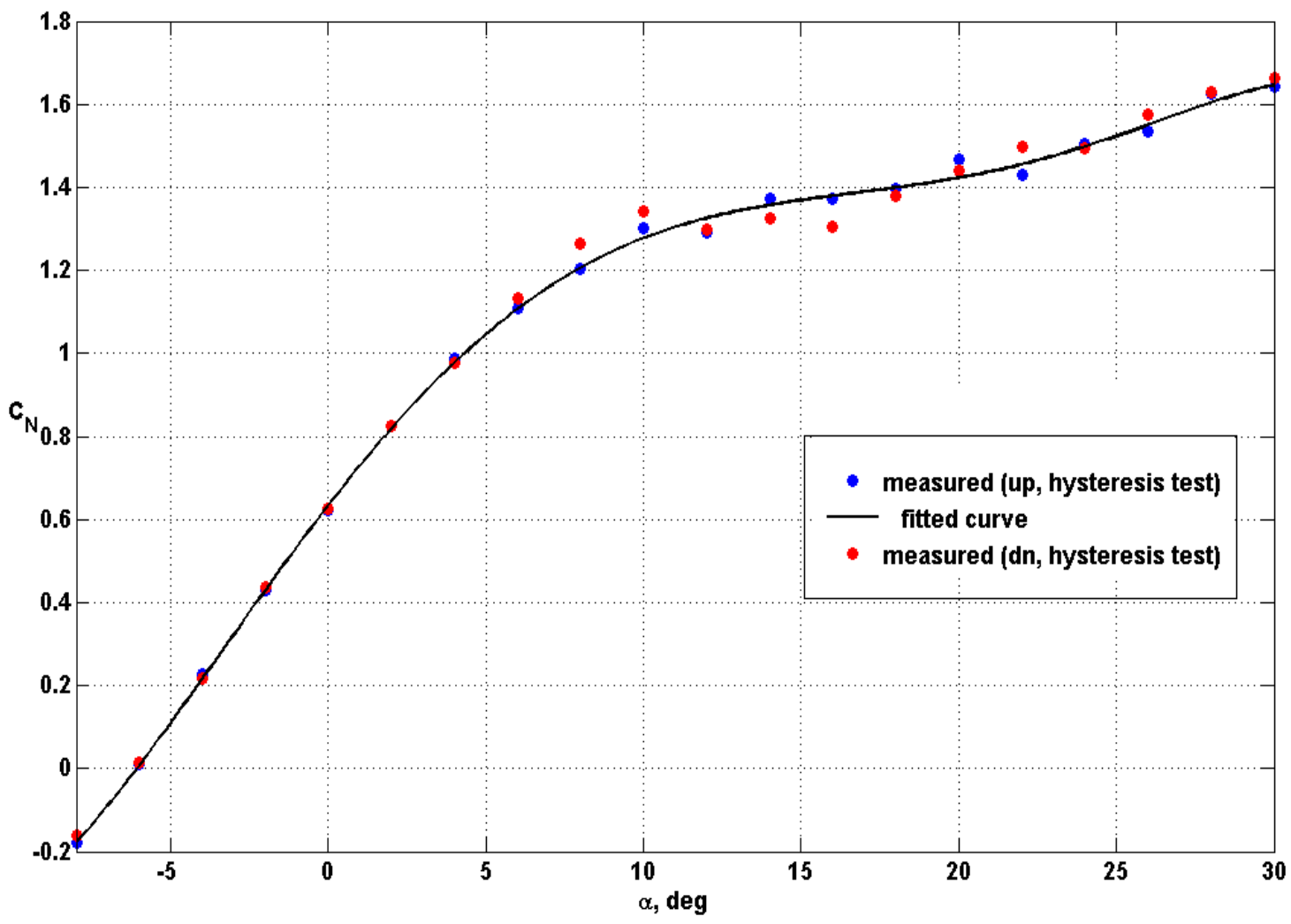

Figure 5. Normal Force as a Function of Angle of Attack at Zero Degrees Sideslip - GL-10 With Motors Off and Control Surfaces, Wing Tilt, and Tail Tilt All Set to Zero Degrees.

If static aerodynamic hysteresis effects were deemed too large to ignore, then the DOE test matrix could have been set up to account for this by including as part of the assessment the direction from which critical test point parameters like angle of attack are approached from the prior test point. (Conceptually this is very similar to how aerodynamic hysteresis effects are assessed using conventional sweeps).

Conventional tests were also used to determine the degree and location of aerodynamic nonlinearities. These tests were necessary to define the appropriate ranges for major factors in the experiment such as angle of attack, sideslip, and motor speed. Another test determined if vortilons were required to improve flight performance in high lift conditions. The vortilons were found to have limited effect and were eliminated from the test matrix. Limited additional OFAT data were obtained to provide representative samples of basic aerodynamics and control effectiveness for later comparison with DOE results.

Given the large test matrix for the main test it was important to determine the minimum sample time that would adequately estimate mean aerodynamic forces and moments in any region of the flight envelope while maintaining statistical accuracy. This sample time study was performed using a DOE test matrix and results are shown in Section VI.

DOE wind tunnel testing in the NASA Langley Low Speed 12-Foot Tunnel required significant changes to the wind tunnel control and data acquisition systems, and some comparisons runs were made early in the GL-10 test to provide confidence that (1) the system changes required for DOE testing had been implemented properly, and (2) the DOE and OFAT traditional sweep testing produced comparable results. This is discussed further in Section VI.

Exploratory tests were used to assess where aerodynamic non-linearities occur for the GL-10, and this was one of the inputs used into determining how to set up blocks of runs. For the GL-10 test, blocking was particularly useful for the following reasons:

1. Flight modes associated with greater non-linearities need to be assessed and processed with higher order terms, whereas flight modes associated with more linear relationships can be assessed with 
lower order terms. Cruise mode run blocks were associated with more linear relationships, whereas Loiter mode run blocks included the more non-linear relationships and interactions associated with flight operations at angles of attack very close to wing stall.

2. In the different vehicle configurations associated with the four modes of flight operation, certain parameters were not active, and there were significantly different limits on the ranges of some parameters. The DOE testing method can characterize the required interactions more efficiently if only the factors capable of being varied are included - and if the ranges are set appropriately. For example, the wing tilt is locked at 90 degrees during Hover - so it is unnecessary to assess all the interactions associated with wing tilt variations when operating in that mode. And during Cruise flight the tail tilt angle range is limited to between -5 and +10 degrees - which makes testing at tail tilt angles up near 90 degrees unnecessary for that vehicle configuration.

3. Physical limitations of the wind tunnel model hardware did not allow for testing across the entire range of vehicle configuration states without making some manual changes to the hardware. The wind tunnel balance being located immediately below the wing tilt pivot axis made it difficult to fit everything within the fuselage contours in that area. This led to a design that required a manual bracket position change to accommodate the full 90 degrees of wing tilt. Transition mode testing was therefore divided up into blocks covering 0 to 45 degrees of wing tilt, and blocks covering 45 to 90 degrees of wing tilt. The prototype flight vehicle has folding propellers that will fold back to reduce drag when the motors are turned off for flight operations in the Cruise or Loiter modes. The wind tunnel model used non-folding propellers, so the Cruise and Loiter mode testing was done with 8 of the propellers physically removed.

\section{Model Hardware Considerations for DOE Testing}

\section{A. Remote Actuation of All Independent Model Parameters}

The requirement of DOE testing to set all the vehicle states and independent parameters to random variations makes remote actuation of all independent model parameters a practical necessity. For the GL-10 wind tunnel model the wing was originally designed to accommodate 6 trailing edge surface actuation servos, 10 electric motors with controllers designed to hold commanded values, and 10 variable pitch propeller actuation servos. As the prototype 50\% scale GL-10 flight vehicle was designed and built, the requirement for the variable pitch propellers was eliminated. Incorporating fixed pitch propellers and propeller speed control on the wind tunnel model allowed the propeller advance ratio to be matched with the prototype flight vehicle.

Depending on the vehicle configuration and modes of operation, there may be individual parameters that are only used for particular flight modes - and this may allow for some parameters to be "fixed" for the corresponding blocks of DOE testing. For example, during testing in the GL-10 Cruise and Loiter configurations the wing tilt was set at a constant zero degrees.

The 6-foot wingspan GL-10 wind tunnel model included control surfaces that could be positioned with commercial off-the-shelf (COTS) digital radio control (RC) model airplane actuation servos. The model propulsion system included COTS RC electric motors, motor controllers, and 9-inch diameter fixed pitch propellers.

The wing tilt used a COTS electric ball-screw actuator, and tail tilt used a custom electric ball screw actuator from a previously tested wind tunnel model. All of the remotely actuated hardware was commanded through hardwire connections to eliminate (1) the need for radio frequency (RF) transmitters and receivers, and (2) potential problems with RF interference.

\section{B. Wiring and Power Requirements}

Since the random setting of motor speeds and control surface positions required constant changes, the wiring, motor controller electronics, and actuation servo electronics all had to be capable of handling the changing currents, signal interference, and thermal loads. Figure 6 is a picture of the wing motor wiring taken early in the model fabrication process. (Note that this does not include any of the control surface actuation servo wiring.)

All the model actuators were electric, and although COTS RC hardware is typically powered by battery packs, power supplies were used for the wind tunnel test. To keep particular sets of motors or actuators isolated and to handle the required current, four different power supplies capable of providing a total of up to 300 amps were used for the model systems. Nine-inch diameter propellers turning at 11,000 rpm pose a significant safety concern, so using independently controllable motor power supplies separate from the supplies for various actuators, allowed for safer checkout and angle calibrations of the control surfaces, wing tilt, and tail tilt. 
Fitting the wire bundles within contour and accommodating up to 90+ degrees of wing or tail tilt changes for hours at a time was also a non-trivial design consideration necessitated by the planned DOE testing.

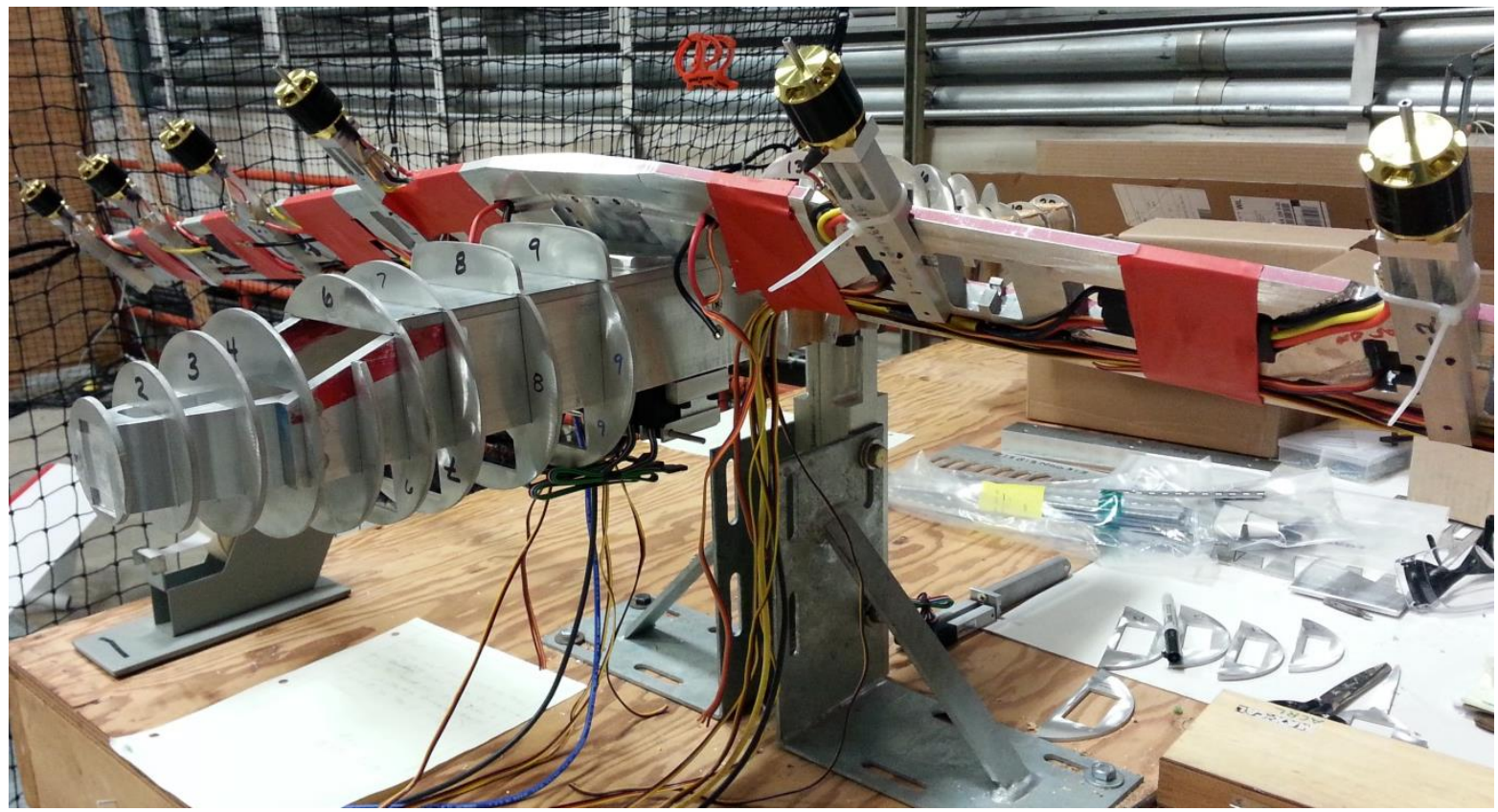

Figure 6. GL-10 Wind Tunnel Model Wing Motor Wiring

\section{Position Sensors and Other Sensors to Measure Motor Speed or Other Commanded Parameters}

Whether sensors providing measured values for the independent parameters are included on the model is a decision typically made well prior to wind tunnel testing, since it can have a significant impact on the design. Figure 7 is a picture of the Free-flying Airplane for Sub-scale Experimental Research (FASER), which is a modified COTS RC model airplane with 7-foot wingspan, a tractor propeller driven by an electric motor, and aerobatic capability. Position sensors were included on FASER, but not on the GL-10 wind tunnel model.

In 2002 FASER was tested in the NASA Langley 12-Foot Low Speed Tunnel, using a combination of traditional sweeps and DOE testing. FASER's lightweight structure (required for flight testing) produced noticeable control surface and linkage deflections during wind tunnel testing. This was anticipated for FASER, and rotary potentiometers were included at each surface to provide measured values for control surface positions that accounted for deflections of the main structure and the actuation servo linkages. 


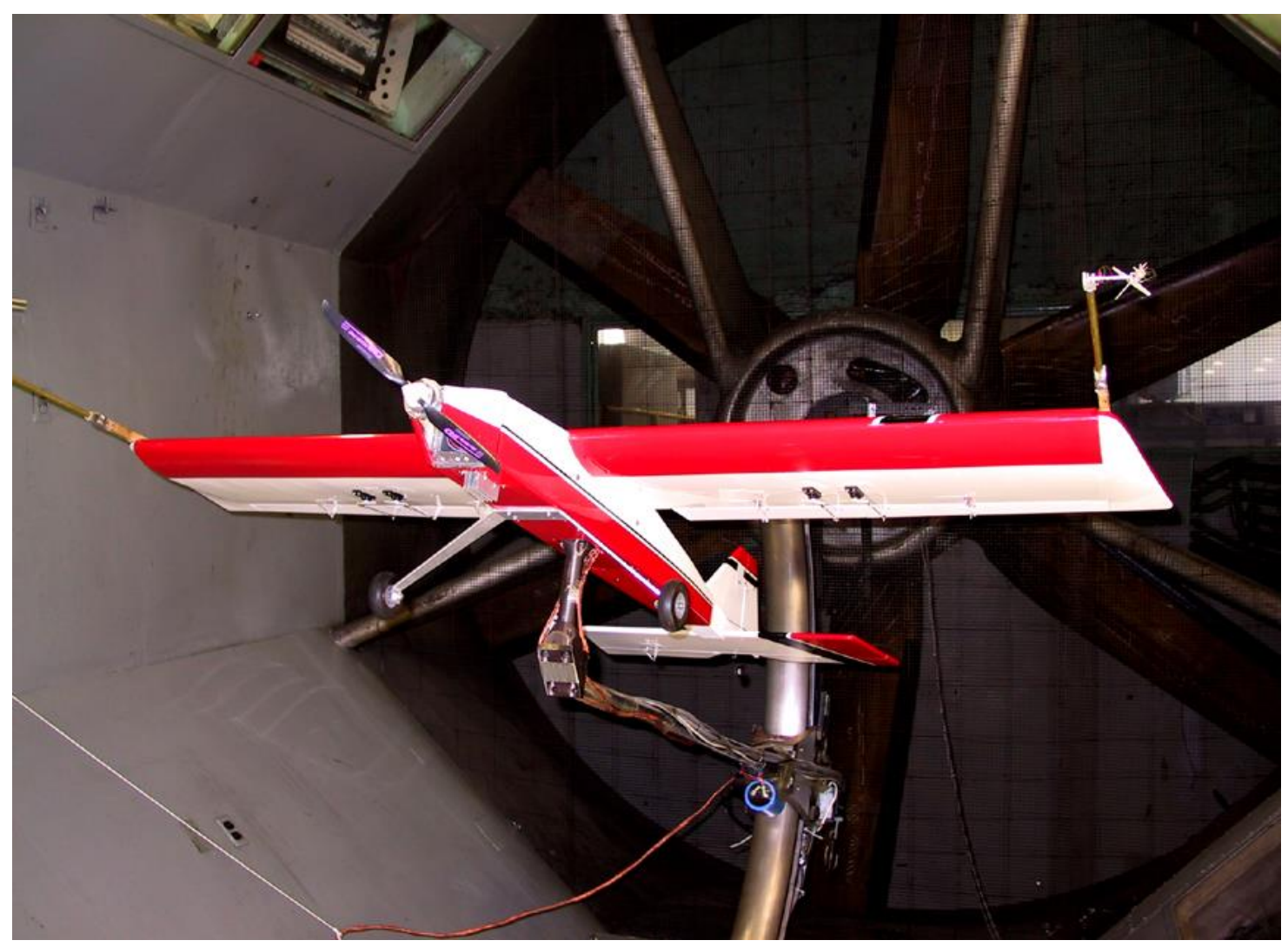

Figure 7. FASER Wind Tunnel Model in the NASA Langley 12-Foot Low Speed

Using sensors to obtain measured position values for control surfaces is a common practice for traditional wind tunnel testing. For traditional OFAT wind tunnel testing there is a tendency to view knowing the actual value for the independent variable as being more important than hitting the original target value. (If you are holding all other variables constant during a sweep, then any value of the independent variable will be located on the same planar curve, defining the functional relationship.) With the DOE approach for this study, each target data collection point can be thought of as being located on a multitude of planar curves defining the functional relationships between all the independent variables - and if the actual values are different from the target for one independent variable, then they will no longer fall on planar curves for the other variables. If slop in the system, structural deflection, linkage deflection or some other factor results in a different value for any of the independent parameters, this will introduce additional error into the data set - even if the actual values have been measured. In DOE terminology this reduces the optimality of the experiment and defeats replication that allows direct estimation of experimental error.

The GL-10 wind tunnel model was built to minimize structural deflections under anticipated test loads. Position sensors were not included for any of the 9 control surfaces for multiple reasons. Without significant shielding the close proximity of sensor wires to multiple electric motor power cables in the wing could cause interference problems. (During bench testing in 2002 this problem was identified for FASER, even though there was only one motor, and it was significantly isolated from most of the wing sensor wiring.)

In addition to potential signal interference concerns, the wiring required for control surface position sensors would have been a significant challenge to fit into the GL-10 wing. For these reasons a different approach was taken for the GL-10 model. The structural stiffness of the model primary structure and the control surfaces was kept high, digital position actuation servos with excellent position accuracy were used, and an upper-lower double link arrangement was used to tie the control surface to the actuation servo. By tensioning the upper and lower links properly, this design allows both links to remain in tension for any control surface position - minimizing link deflection and preloading against any slop in the mechanisms. Figure 8 is an in-work fabrication photo showing this arrangement before the servo arms were trimmed back. Subsequent bench test measurements showed good agreement between commanded and actual control surface positions. 


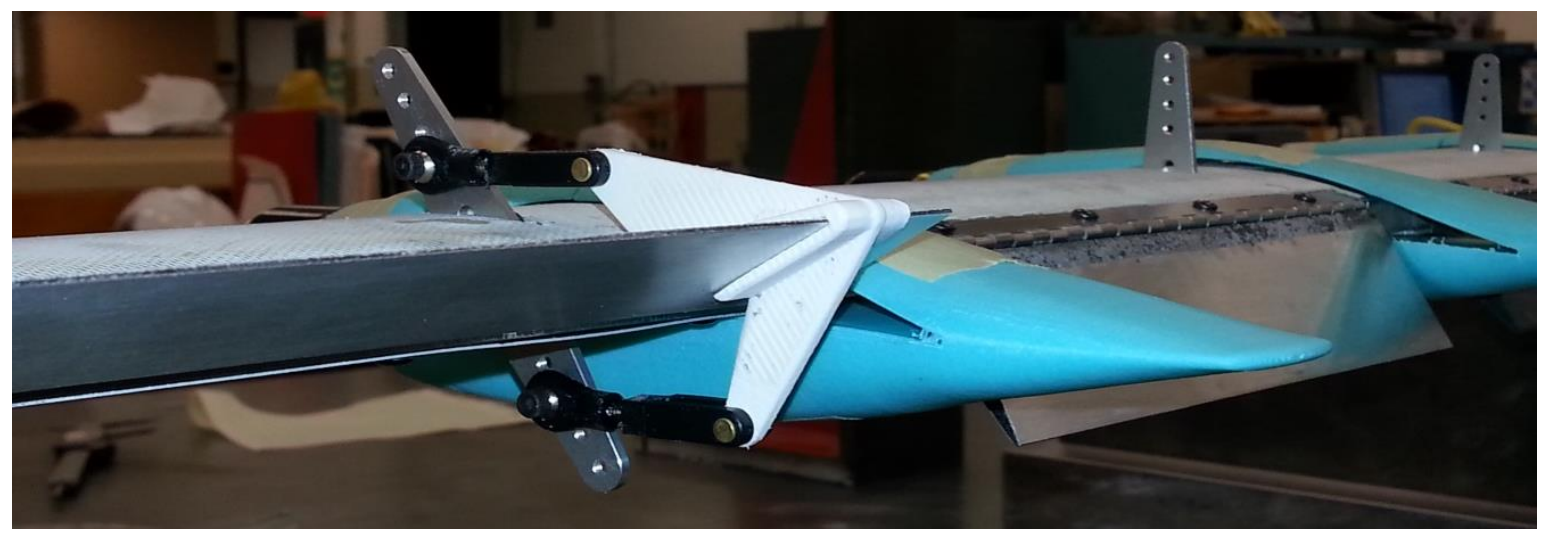

Figure 8. GL-10 Control Surface Double Link Actuation

\section{Cautions When Using Commercial Off-the-Shelf (COTS) Radio Control (RC) Hardware}

The performance of COTS RC motors, motor controllers, and actuation servos has significantly improved in recent years - making their incorporation into reduced-scale wind tunnel models a cost effective option when propulsion or remote actuation is required, The structural and aerodynamic loads imparted to COTS RC hardware during wind tunnel testing will typically be much higher than what the same configuration would see during flight testing, and the loading on particular components in the tunnel will typically be acting for much longer periods of time. The impact of the higher loading and the need to hold particular positions or power settings for longer periods of time needs to be assessed carefully during the model design and fabrication process.

For the 2002 FASER DOE testing the basic empennage structure of the RC model kit had to be stiffened and strengthened with fiberglass to keep it from breaking, and to reduce deflections. When an RC airplane experiences a large control surface deflection or attitude change in flight, the aerodynamic forces are primarily reacted by the inertia of the airframe. Typically the inertia is not sufficient to react all of the aerodynamic forces, and the airframe immediately starts moving. In a wind tunnel an RC airplane with large control surface deflections or at a large angle of attack or sideslip can react the full magnitude of the aerodynamic forces because it is mounted to a model support system that typically keeps it from moving to relieve the load.

Rotary position pots for control surfaces were definitely needed on FASER. The higher aerodynamic loading in the wind tunnel exceeded the RC actuation servo's ability to hold commanded position for certain test conditions, and produced excessive control linkage deflection that degraded the position accuracy. The electric motor and motor controller performance was acceptable for flight testing limited to several minutes by the available battery power. But for longer testing periods on the bench or in the wind tunnel using power supplies, motor and motor controller reliability was a significant problem - requiring multiple replacements over the course of bench and tunnel testing.

For the GL-10 DOE test the digital control surface actuation servo and linkage design described in Section IV C performed well, with absolutely no problems over the course of several weeks of testing. The electric motors and motor controllers were selected so that they would be operating well below their continuous speed and power ratings. The only overheating problem encountered was due to a bad electrical connection, which was quickly fixed. There was a significant problem with the motors initially - and this was ultimately identified as being the result of the higher aerodynamic moments acting on propellers held at very high angles of attack for long periods of time. Motor shaft bearing tolerances for the COTS motors were adequate for the lower off-axis moments experienced momentarily during free flight, but allowed the outer case to make local contact with the stator during wind tunnel testing. Fortunately a relatively minor modification to the case for all the motors solved the problem. During the final week of testing the motors ran for approximately six hours per day with no problems.

Although RC motor controllers have been improving in reliability, our experience has been that their ability to hold a particular speed can be degraded by a variety of factors, including motor wear and performance changes over the period of use. We would therefore strongly recommend using additional sensors to measure motor speed whenever possible - particularly since deviations from the commanded (desired) values are not visually apparent.

During bench testing of COTS RC actuators originally selected for the wing and tail tilt mechanisms, the mechanical slop in the actuators was measured to be seven times the value listed on the product specification sheet. (According to one of the main suppliers for these actuators, the ones originally developed in the US were held to 
tighter tolerances. When manufacturing was sent overseas, the tolerances were loosened, but the product sheet was never changed.) Although modifications to the actuator could improve things some, the resulting performance was ultimately deemed unacceptable and the wing and tail tilt actuators were replaced prior to starting the main GL-10 DOE runs.

\section{E. Actuation Rates vs. Actuation Ranges}

The randomization associated with DOE testing requires all the independent parameters being assessed during a block of runs to change between each data collection point. The time it takes to get all the independent parameters to their commanded values is typically a function of the actuation rate and the magnitude of the change between test point values. For the GL-10 DOE test the tail tilt actuation was the parameter that ultimately determined how quickly data could be taken. The tail tilt actuation rate was no slower than the rates for certain other parameters (including tunnel angle of attack and sideslip), but the deflection range for tail tilt in most of the flight modes was 2 to 3 times greater than any other parameter - with the result being that after all other parameters had reached their next data point value, the tail tilt was still moving. Assessing the rates of change vs. ranges for all independent parameters of a planned DOE test may identify particular parameters where hardware changes to increase actuation rates can significantly reduce the overall testing time required.

\section{NASA Langley 12-Foot Low Speed Tunnel Hardware, Software, and Data Acquisition Development Enabling DOE Testing}

Figure 9 is a cutaway of the NASA Langley 12-Foot Low Speed Tunnel showing some of the major features of this facility. Reference 7 provides overview of dynamic test techniques used at NASA Langley Research Center on scale models to obtain a comprehensive flight dynamics characterization of aerospace vehicles - including testing in the 12-Foot Low Speed Tunnel.
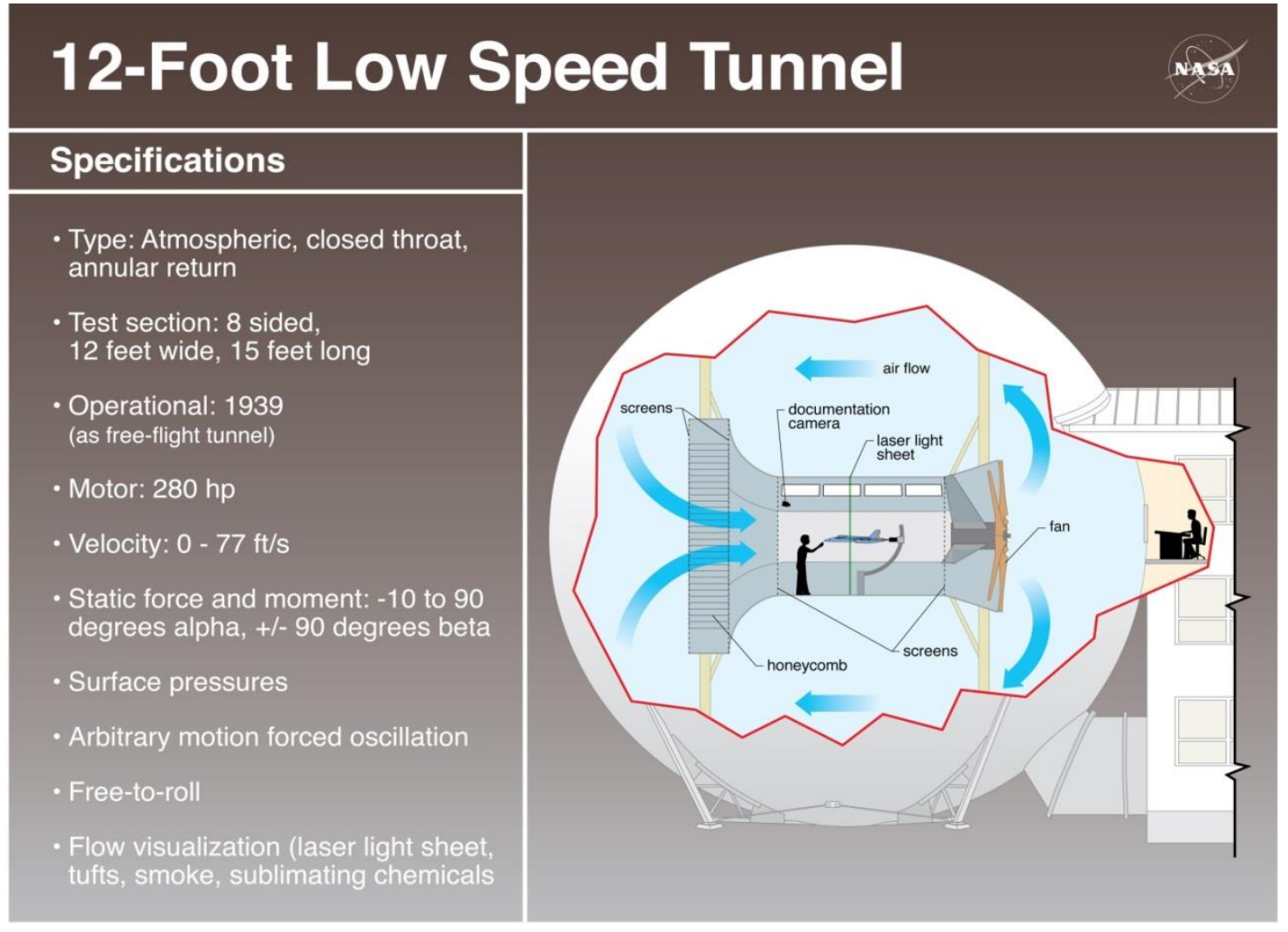

Figure 9. Facility Cutaway for the NASA Langley 12-Foot Low Speed

Models for static testing in the NASA Langley 12-Foot Low Speed Tunnel are typically mounted on a sting attached to a C-strut. The sting may attach directly to the model - or the model may be mounted on a shorter vertical 
post attached to the end of the sting. A vertical post mount was used for the FASER testing (Fig 7) and for the GL10 test (Fig 10). The aft end of the sting is moved along the C-strut to change the angle of attack from -10 degrees up to +90 degrees while keeping the model generally centered in the tunnel. The entire C-strut is rotated about a vertical axis to change the angle of sideslip between -90 and +90 degrees. The C-strut also has a vertical translational range of travel of 2 feet.

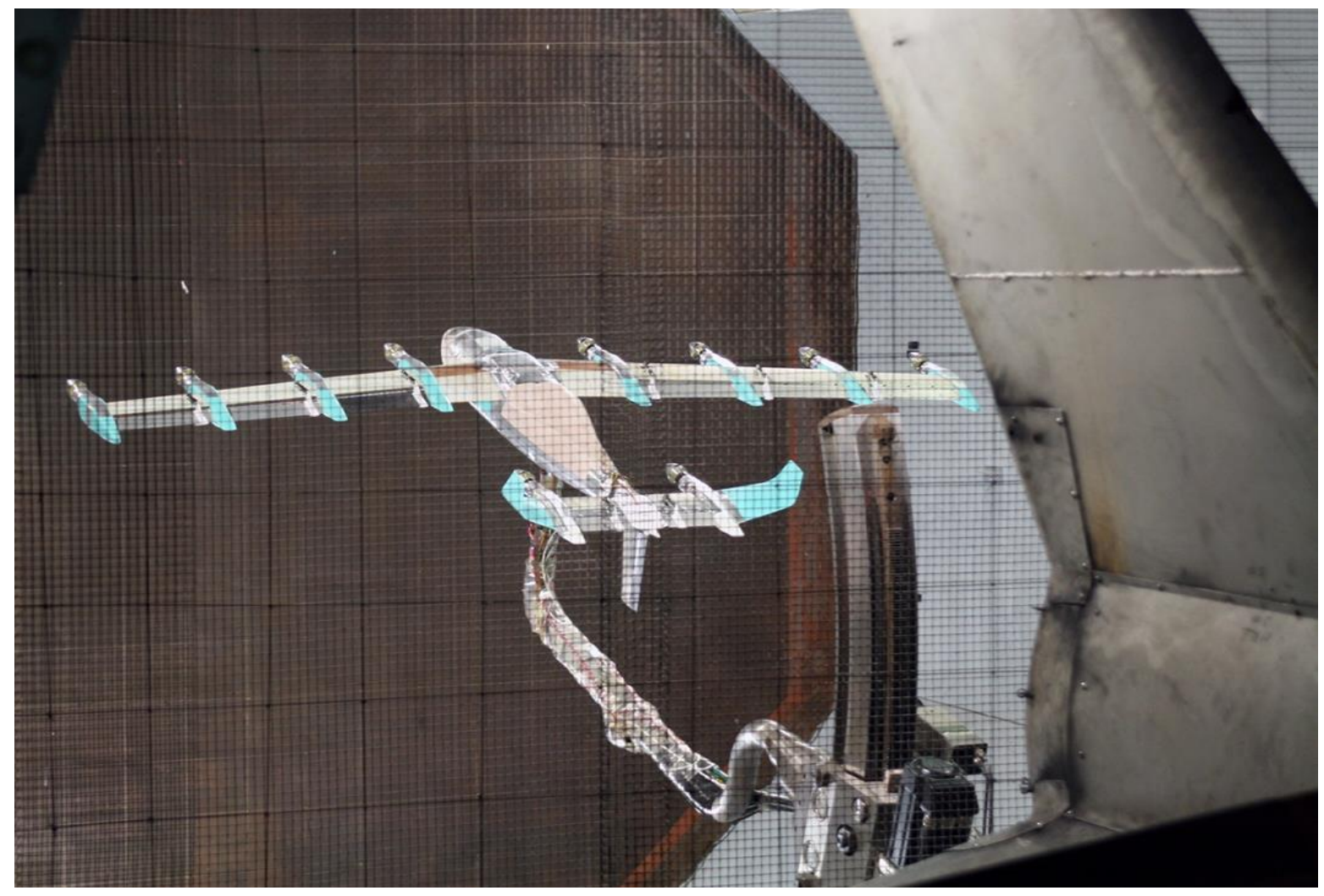

Figure 10. GL-10 Wind Tunnel Model in the NASA Langley 12-Foot Low Speed

In 2002 when the FASER DOE testing was done at the 12-Foot Tunnel all the model parameters were remotely set through a dedicated laptop computer hooked directly to the model system, and not by the tunnel control system. At that time the tunnel control software for setting angle of attack and sideslip was configured to handle traditional sweeps, and could not accommodate random positioning movements within a single run. For the 2002 FASER test this required that each data point be obtained as a separate tunnel run. This complicated the post-test processing of the data - although it was doable because there were only 7 independent variables.

The amount of tunnel support system movement during DOE testing will typically be much greater than for more traditional OFAT testing, and there was some initial concern about the potential for increased wear or damage to the facility equipment if extensive DOE testing was done. The mechanical slop, hysteresis, and actuation rates for the facility actuators moving the model support system to set angle of attack and sideslip will also have a direct impact on the DOE test efficiency, and the accuracy of the data collected - so this was also a concern for the researchers.

Actuator upgrades since the 2002 FASER DOE testing coupled with tunnel control software changes have significantly improved the facility's capability to perform DOE testing efficiently without damaging equipment. For the 2013 GL-10 test individual tunnel runs typically contained between 190 and 330 individual static test points. Commanded values for all 23 independent parameters were set at each test point from a single input file in the tunnel control system. All independent parameter commanded values, all measured data from the 6-component strain gage tunnel balance inside the model (averaged over the data sampling time), and any position sensor measured values were recorded for each test point, and output in a single file - greatly simplifying the postprocessing of the data after the test. (Section VI describes how the data sampling time was determined for the GL-10 test.) 
Vehicles that undergo significant changes in the center of gravity (CG) position present a challenge for DOE testing. The wing tilt and/or tail tilt of the GL-10 will change both the longitudinal and vertical position of the CG, and therefore weight tare effects will not remain constant over the course of a single DOE run. Traditional OFAT testing typically involves making alpha \& beta sweeps with the wind tunnel model hardware in a fixed configuration - so the CG does not change over the course of a run.

For the GL-10 DOE test, tares were measured on a point-by-point basis. A DOE tare "run" consisted of a windoff motors-off run through the entire DOE block of points - recording the 6-component balance gravity vector force and moment components with all independent parameters (other than motor speed) set to their test point values. Each DOE run test point therefore had a unique set of tare values. The tunnel data acquisition and processing system applied these tares to the corresponding data points for the subsequent wind-off-motors-off and wind-on-motors-on DOE runs. This was a significant change to the tunnel data processing software - but it was key to enabling DOE testing on complex vehicle configurations like the GL-10.

For the GL-10 DOE testing the four types of "runs" listed below could be made. The sequence and commanded values for all independent variables (except motor speed) at each test point would be the same for all four runs.

Run A. Wind Off, Motors Off

Run B. Wind Off, Motors On

Run C. Wind On, Motors On

Run D. Wind On Motors Off

The tare run is required - since the data obtained from this run is subtracted from all the other runs to eliminate the model gravity vector force and moment components. The following list indicates the type of forces and moments that can be determined using the data from various combinations of Runs A, B, C, and D.

Run A = Gravity vector (tare) forces and moments

Run B - Run A = Propeller thrust-induced forces and moments

Run $\mathrm{C}-$ Run $\mathrm{A}=$ Airframe aero plus propulsion-induced forces and moments

Run D - Run A = Airframe aero forces and moments without propulsion-induced aero influences

Run C - Run B = Airframe aero forces and moments in the presence of propulsion-induced aero influences

Since the net aerodynamic force and moment data in the presence of propulsions effects is of higher priority to the prediction of flight dynamics, a decision was made to only do Runs A, B, and C for the GL-10 test.

The GL-10 test was a static test, with data only being recorded at the specific test points. It is therefore important to consider how to determine when the system should take data. For DOE static test runs the amount of travel time required to get from one test point (with all parameters at their commanded values) to another will vary as a function of the change in individual parameter values from the previous point and the corresponding actuation rates (or speed change rates). Using an average value will not work, since acquiring data before all parameters are set will obviously produce bad data. Using a sufficiently large constant time increment to ensure all parameters are set for any test point change is very inefficient, since the travel times between the majority of test points will be significantly less than this.

In the months prior to the GL-10 test, another DOE test was done on a Kahu model. For that test a particular COTS Maestro interface/control board was used to set independent parameters on the model, and software was written to send a signal to the tunnel data acquisition system when the model parameters were set and data could be taken. Recognizing that this work was being done for the Kahu DOE test, a decision was made to use the same hardware and software for the GL-10 test.

In Section IV it was noted that for the GL-10 wind tunnel model the tail tilt actuation consistently took the longest amount of time to get from one test point value to the next test point value. A minimum delay sufficient to cover all other parameters reaching their commanded positions was combined with a measured position from a potentiometer on the tail tilt actuator to determine when the model parameters were set, and a signal was sent to the tunnel system indicating that data could be taken. This arrangement allowed data to be taken as efficiently as possible.

\section{Examples of GL-10 Modeling Results}

A key goal early in the GL-10 study was to reduce the time required to obtain each data point. Prior to this test, conventional practice for the 12-Foot Tunnel was to sample each test point for a set period of time, typically 10 
seconds, and use the averaged values. An exploratory test (nested FCD) considering sample times of 2, 4, 6, 8, and 10 seconds was performed to determine the fastest sample time with adequate accuracy. Shown in Fig. 11 are the normal force measured values (red dots) and the values computed from the mathematical model (black line). This result is also typical of the other aerodynamic coefficients. Measured values were obtained for the range of sample or dwell times and over a broad range angle of attack and sideslip. The results show no statistical difference for a dwell time of 2 seconds versus a dwell time of 10 seconds. The "I-bars" are the least significant difference (LSD) at the $95 \%$ confidence level. Because the bars for the 2 -second and 10 -second dwell times overlap no statistical difference in dwell times is observed.

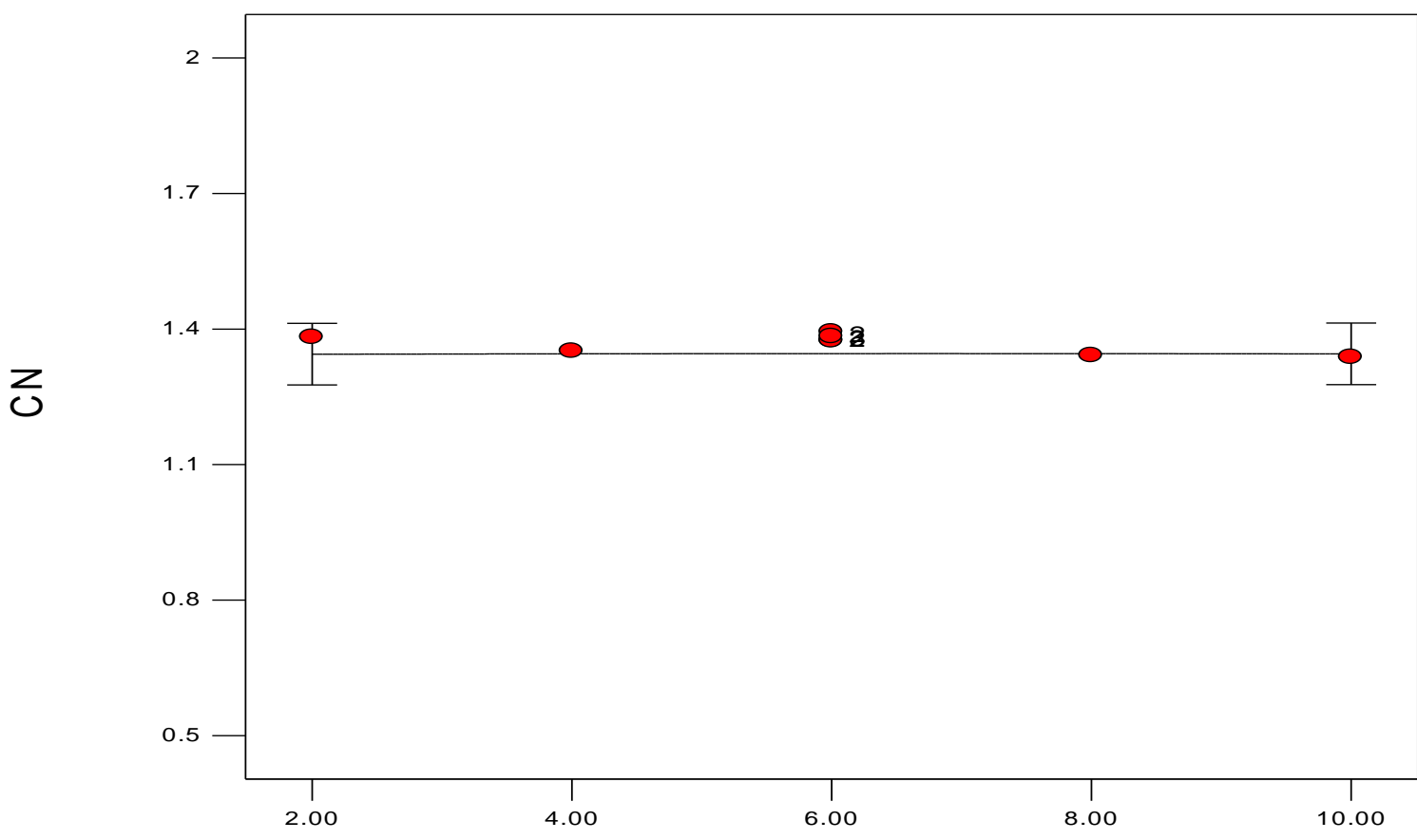

C: dwell time (s)

\section{Figure 11. Normal Force as a Function of Sample Time - GL-10 with Motors Off and Surface Deflections Set to Zero Degrees}

A convenient way to confirm this result is to view the normal force as a function of angle of attack estimated with data using the two extreme values of dwell time. Figure 12 shows the computed curves that correspond to a dwell time of 2 seconds as a black line, and a dwell time of 10 seconds as the red line. No visible difference exists between the normal force curves, confirming no statistical difference in the dwell times. 


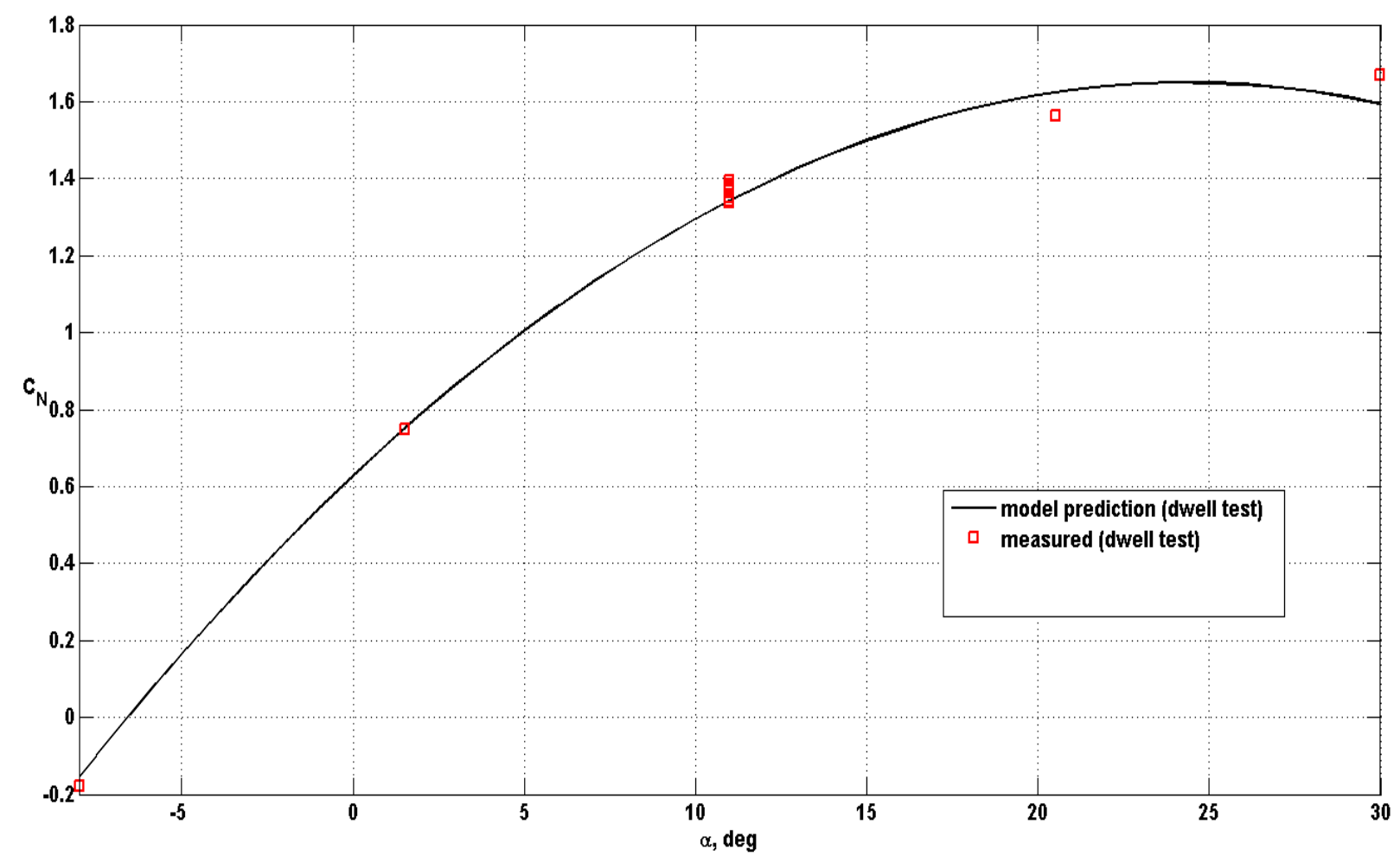

Figure 12. Normal Force as a Function of Angle of Attack, for Two Values of Dwell Time - GL-10 with Motors Off and Surface Deflections Set to Zero Degrees

If the DOE-generated plots for $\mathrm{CN}$ vs. dwell time are co-plotted with the static aerodynamics hysteresis $\mathrm{CN}$ sweep data shown in Fig. 5, this provides the comparison between DOE and sweep results shown in Fig. 13. After assessing the static aerodynamic hysteresis based on sweeps, a decision was made that the hysteresis could be ignored for the DOE testing. Therefore Fig. 13 does not include hysteresis effects for the DOE results. The curve generated by DOE deviates significantly from the sweep data for the higher angles of attack. This is a result of the sparsity of DOE points taken. (The DOE run was not originally designed to capture the detail of the CN relationship to angle of attack.)

Figure 14 shows the DOE aerodynamic model for $\mathrm{CN}$ based on data covering Cruise-to-Loiter Mode with engines running from 4000 to $12000 \mathrm{rpm}$. The measured data is taken from a portion of the sweeps data used in checking for hysteresis without engines. For this example the model prediction curve was based on the following factor settings: beta $=0$ degrees, all surfaces $=0$ degrees, two outboard engines idle at $4000 \mathrm{rpm}$, wing tilt $=0$ degrees and tail tilt $=0$ degrees. The blue and red markers show the measured data points from the upward (increasing) and downward (decreasing) alpha sweeps done to check for static aerodynamic hysteresis. In comparison to the Fig. 13 plot, the Fig. 14 curve generated from a greater density of DOE test points does a better job of capturing the non-linear character of the normal force at the higher angles of attack - and more closely follows the data taken during the hysteresis check. 


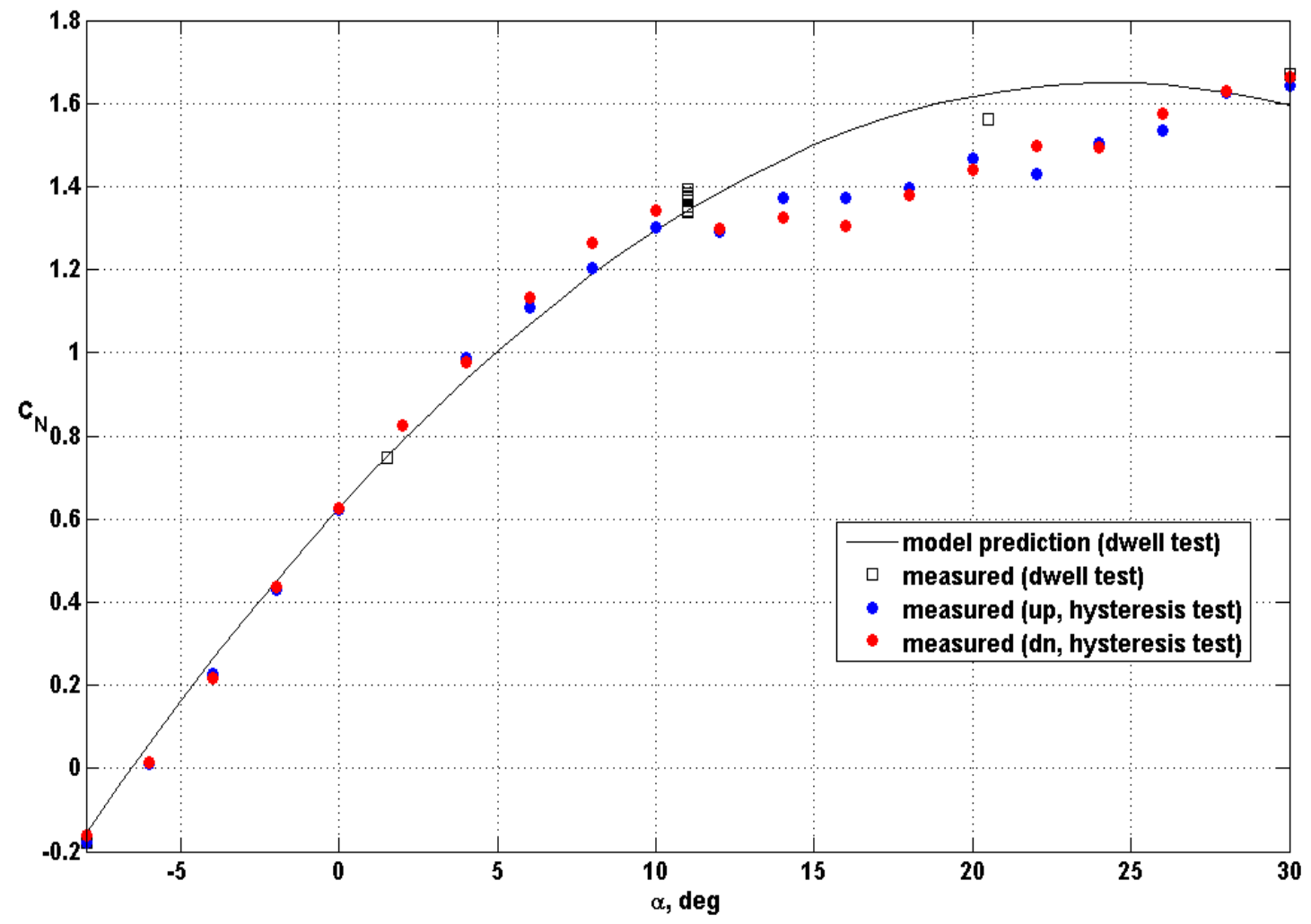

Figure 13. Normal Force as a Function of Angle of Attack, for DOE Test for Dwell Time, and Sweep Data for Hysteresis Check - GL-10 with Motors Off and Surface Deflections Set to Zero Degrees 


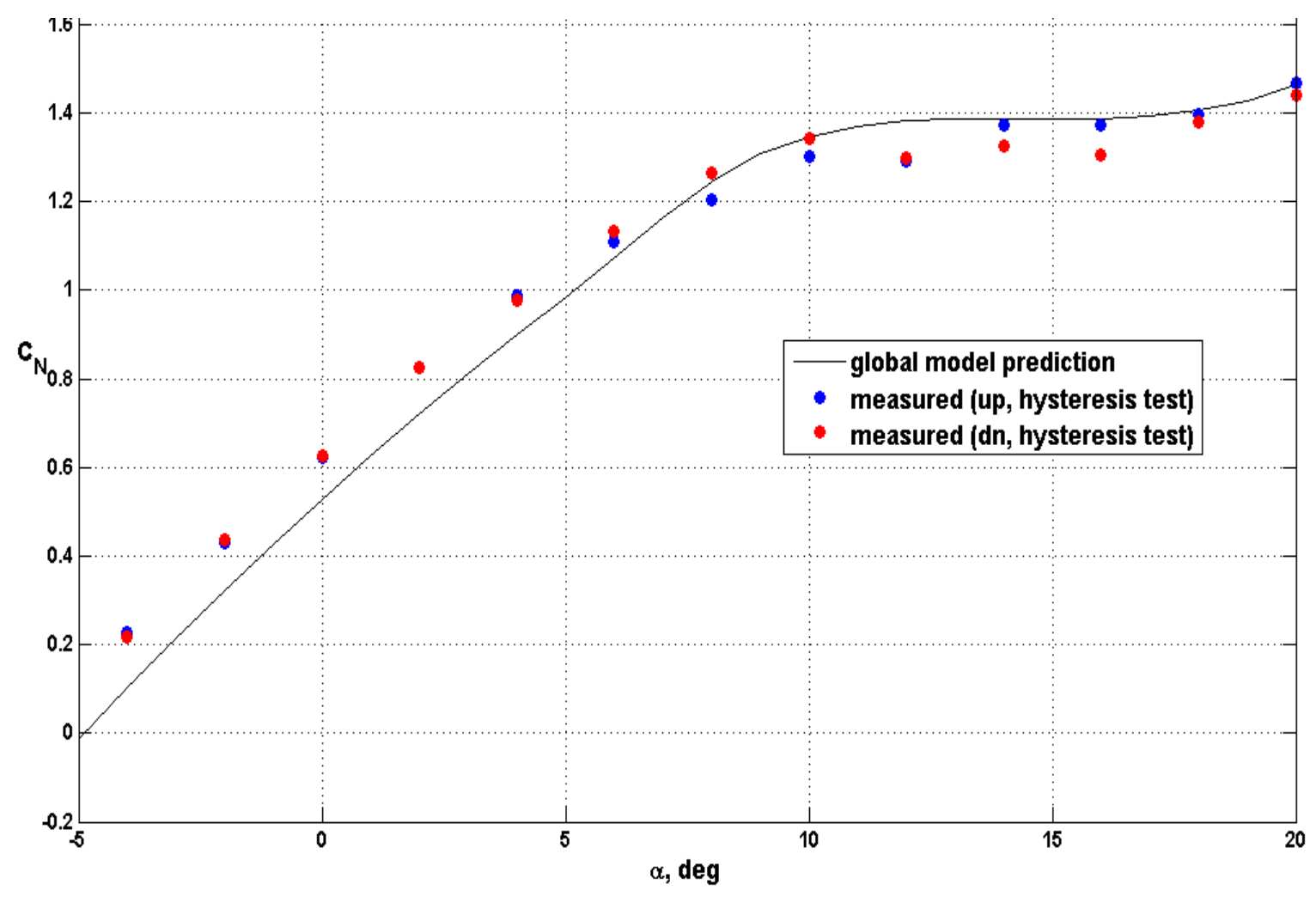

\section{Figure 14. Normal Force as a Function of Angle of Attack, for DOE Test for Cruise-to- Loiter Mode, and Sweep Data for Hysteresis Check - GL-10 with Motors Off and Surface Deflections Set to Zero Degrees}

In Cruise mode the GL-10 operates as a conventional aircraft. Wing tilt is not used, tail tilt is limited, and only two outboard motors provide propulsion. This leaves only 14 factors for this mode to be tested. Loiter mode is configured the same as the Cruise mode configuration but in this mode GL-10 operates closer to the stall angle of attack. In transition mode all 23 factors are used in the design. In Hover mode the factors are reduced to 19.

As an example of the DOE designs for GL-10, design test points are shown for the Cruise mode in Fig. 15. This is a representative set of test points shown in terms of angle of attack and sideslip. The limited ranges for angle of attack $\left(-5^{\circ}\right.$ to $\left.10^{\circ}\right)$ and sideslip $\left(-5^{\circ}\right.$ to $\left.5^{\circ}\right)$ reflect the expectation of a conventional flight envelope in this mode. Similar graphics were obtained for any combination of factors considered. The four colors black, red, green, and blue, correspond to the test blocks 1 to 4 , respectively. Blocks 1 and 2 define a nested FCD and blocks 3 and 4 are the optimized test points that minimize prediction error.

For the Cruise mode, modeling work suggested that a quadratic model for normal force is adequate. This model covers all main effects, 2-factor interactions, and the quadratic behavior of normal force with angle of attack. A surface plot showing the estimated normal force model as a function of angle of attack and tail-tilt (Ttilt) is shown in Fig. 16. Measured values are presented on the chart as red dots if they are above the predicted surface and pink dots if they are below. Limited measured values are shown for clarity. Five measured values are shown at the center of the range for each independent factor. The remaining factors were set to their mid-range values to create this surface. The factor values are shown in the legend. 


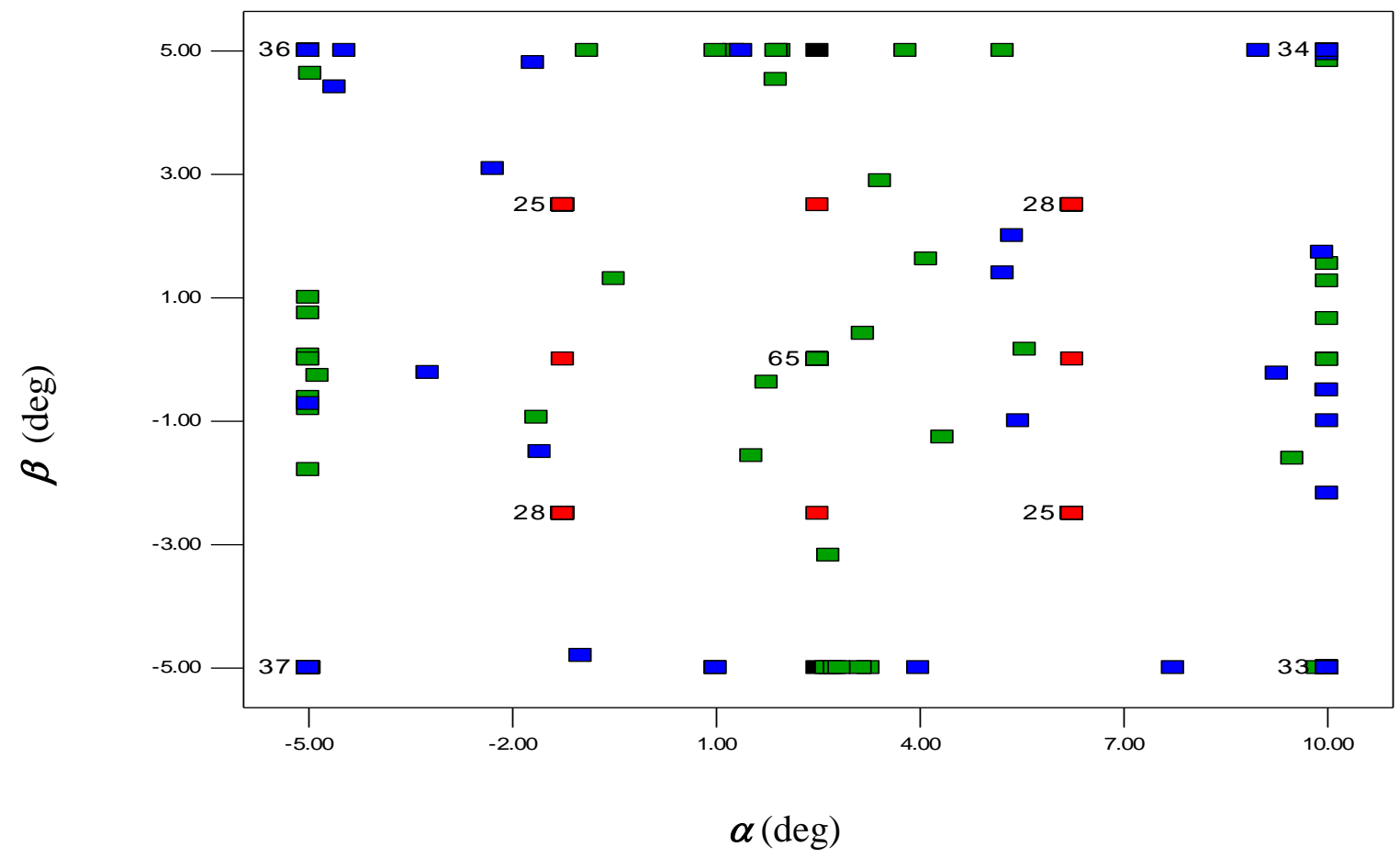

Figure 15. Nested Face-Centered Design with Optimized Test Points

Design-Expert $\AA^{\circledR}$ Software

Factor Coding: Actual

- Design points above predicted value

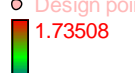

$-0.611523$

$\mathrm{X} 1=\mathrm{A}:$ aoa $\mathrm{X} 2=\mathrm{L}:$ Ttilt

Actual Factors

B: beta $=0.00$

C: LWS1 $=-7.50$

D: LWS2 $=-7.50$

$\mathrm{E}:$ LWS3 $=-7.50$

F: RWS4 $=-7.50$

G: RWS5 $=-7.50$

$\mathrm{H}:$ RWS6 $=-7.50$

$\mathrm{J}: \mathrm{LTS} 1=-7.50$

$\mathrm{K}: \mathrm{RTS} 2=-7.50$

$\mathrm{M}: \mathrm{Rud}=0.00$

$\mathrm{N}$ : LWE1 $=8000.00$

O: RWE8 $=8000.00$

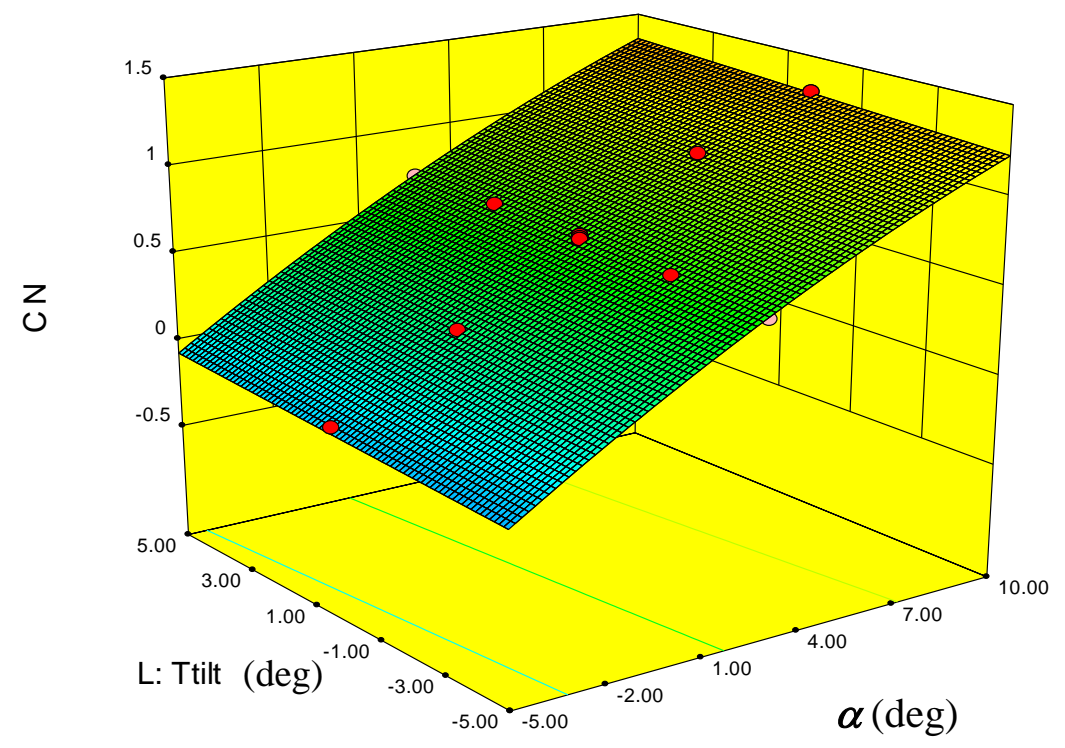

Figure 16. Normal Force as a Function of Angle of Attack and Tail-Tilt in Cruise Mode 


\section{VII. "Learn-to-Fly" Test Development}

All of the DOE wind tunnel tests previously discussed in this paper were static tests, with data only being taken after all parameters reached the commanded values. As part of ongoing "Learn-to-Fly" efforts, researchers at NASA Langley are developing methods to take continuous data and have the software assess the results to characterize the aerodynamic relationships between critical vehicle parameters in near-real-time. The intent of Learn-to-Fly efforts is to autonomously develop processes that will characterize the flight dynamics of a vehicle up through and including the ability to achieve the desire flight capability with minimum human interaction and time. The ability to rapidly update simulation tools (either aerodynamic models, or flight response models) based on flight testing is the ultimate goal - although the process is being developed in conjunction with and also has application to wind tunnel testing. Learn-to-Fly will ultimately include System Identification methods (Ref. 9), feedback control, and automated experiment design. One high level conceptual approach for how this could be done is described below:

1. Using feedback from forces and moments (for rigidly mounted models) or from accelerations and rates (for models free to rotate about one or more axes) to trim the vehicle to zero out moments and/or rotations.

2. Commanding control surface or thrust perturbations from trim and collecting initial data to characterize the primary aerodynamic responses. Conceptually this is like the exploratory tests done for DOE involving static test points - and it serves a similar function in helping the software define the additional combinations of commanded perturbations to be tested.

3. Additional data continues to be collected, with the commanded perturbations being varied as the vehicle aerodynamic response characteristics and the interactions between control parameters are further refined. If the model is free to move (i.e. is a flying model or is mounted on a "free-to-roll" rig) the response characteristics can include inertial forces and moments if sensors to measure inertial forces are part of the data collection set-up for the test.

Learn-to-Fly efforts to take continuous data with commanded values for independent variables being developed in near-real-time presents a number of challenges for wind tunnel testing - particularly if the vehicle goes through significant configuration changes. For example, during the GL-10 static DOE testing, the tare values for each test point were determined by a separate run with the wind off and all propeller motors off. This approach works when the exact sequence of test points is known beforehand. For the Learn-to-Fly testing, this is not the case - since the commanded positions for control surfaces or throttle settings will only be determined as data is being gathered.

One solution for this in a wind tunnel is to do a wind off static DOE test with the sole purpose of characterizing the tare values as a function of the other independent parameters. For the GL-10 test the previously measured tare values at each test point were subtracted from the values at the corresponding test points on a one-to-one point-bypoint basis for the actual data runs. But theoretically the same tare run data could be used to develop a "tare model" that could be used to predict the appropriate tare value for any combination of the other independent parameters. This would allow the appropriate tares to immediately be applied during wind-on runs with or without thrust. Some variation of this approach is necessary for Learn-to-Fly wind tunnel testing - but it could also be used to more efficiently conduct static DOE tests.

\section{Concluding Remarks}

Some key contributions to the application of the DOE approach to wind tunnel testing are included in Refs. 3 to 6. Reference 3 more fully describes the 2002 FASER DOE testing in the NASA Langley 12-Foot Low Speed Tunnel discussed in Sections IV and V. Reference 5 describes application of DOE to the Boeing Blended Wing Body configuration. In that case numerous surfaces were ganged together, reducing the total number of factors (independent variables) to eight. An efficient fractional factorial design was created to capture main effects, as well as the two-factor and three-factor interactions. In DOE "factorial designs" require a certain number of runs to fully meet the estimation and statistical requirements. A "fractional factorial design" is a factorial design with fewer runs (a carefully chosen subset) that retains certain desirable statistical characteristics to allow the main features of the relationships between variables to be determined.

Reference 6 describes a nested-face-centered design (FCD) that was created for the X-31 aircraft with 5 factors. This novel experiment design incorporated the advantages of a fractional FCD and ensured 5 equal levels for control surface deflections that cover the full range of motion. Given the complex nature of the GL-10 vehicle configuration with up to 23 factors, the current study extended the previous work by blending nested FCD designs that minimize the integral of prediction error across the design space. Reference 8 includes a description of the ongoing effort to 
use the data obtained from the GL-10 test to develop simulation models and control laws for the prototype flight vehicle.

As described in Section IV and V, the ability to do DOE wind tunnel testing with larger numbers of factors imposes significant requirements on the wind tunnel model hardware, test facility hardware, control software, and data acquisition systems. Facility hardware and software upgrades to the NASA Langley 12-Foot Low Speed Tunnel since the initial DOE tests of FASER in 2002 were essential for enabling the recent GL-10 DOE testing. Increased awareness of the practical implications of DOE testing during wind tunnel system upgrades or equipment replacement is needed if the tremendous benefits of DOE are to be more broadly realized for wind tunnel testing at other facilities.

\section{Acknowledgments}

The GL-10 work discussed in this paper was conducted under the NASA Reimar Project, with contributions provided by individuals from multiple branches at NASA Langley Research Center. The authors would also like to thank the Subsonic Fixed Wing Program for supporting some of the recent test technique development effort at the NASA Langley 12-Foot Low Speed Tunnel.

\section{References}

${ }^{1}$ Fredericks, William J., Moore, Mark D., and Busan, Ronald C. "Benefits of Hybrid-Electric Propulsion to Achieve 4x Cruise Efficiency for a VTOL UAV,” 2013 International Powered Lift Conference. August 2013.

${ }^{2}$ Montgomery, Douglas, C., "Design and Analysis of Experiments," $8^{\text {th }}$ ed.,Wiley, 2013.

${ }^{3}$ Morelli, E.A. and DeLoach, R. "Response Surface Modeling using Multivariate Orthogonal Functions," AIAA paper 20010168, $39^{\text {th }}$ AIAA Aerospace Sciences Meeting and Exhibit, Reno, Nevada, January 2001.

${ }^{4}$ Morelli, E.A. and DeLoach, R. "Wind Tunnel Database Development using Modern Experiment Design and Multivariate Orthogonal Functions," AIAA paper 2003-0653, $41^{\text {st }}$ AIAA Aerospace Sciences Meeting and Exhibit, Reno, Nevada, January 2003.

${ }^{5}$ Landman, Drew, Simpson, Jim, Vicroy, Dan D., and Parker, Peter, "Response Surface Methods for Efficient Complex Aircraft Configuration Aerodynamic Characterization,” Journal of Aircraft, Vol. 44, No. 4, July-August 2007.

${ }^{6}$ Landman, Drew, Simpson, Jim, Vicroy, Dan D., and Parker, Peter, "Hybrid Design for Aircraft Wind-Tunnel Testing Using Response Surface Methodologies,” Journal of Aircraft, Vol. 44, No. 4, July-August 2007.

${ }^{7}$ Owens, D. Bruce, Brandon, Jay M., Croom, Mark A., Fremaux, C. Michael, Heim, Eugene H., and Vicroy, Dan D. "Overview of Dynamic Test Techniques for Flight Dynamics Research at NASA LaRC (Invited)," AIAA paper 2006-3146, 25th AIAA Aerodynamic Measurement Technology \& Ground Testing Conference, San Francisco, CA, June 6, 2006

${ }^{8}$ Rothhaar, Paul M., Murphy, Patrick C., Bacon Barton J., Gregory, Irene M., Grauer, Jared A., Busan, Ronald C., and Croom, Mark A.. "NASA Langley Distributed Propulsion VTOL Tilt-Wing Aircraft Testing, Modeling, Simulation, Control, and Flight Test Development," $14^{\text {th }}$ AIAA Aviation Technology, Integration, and Operations Conference, Atlanta, GA, June 2014.

${ }^{9}$ Klein, Vladislav and Morelli, Eugene, "Aircraft System Identification: Theory and Practice," 1st edition, AIAA Inc., Reston, Virginia, 2006. 